\title{
Editorial
}

\section{Atracurium, pharmacokinetics and metabolites}

The pharmacology applicable to anaesthetic practice is often different from that of other specialities. The drugs used are different, a short duration of action is desired to increase flexibility and toxicity is not an overwhelming concern because of the short duration of exposure. Thus, recent drug development has focused on short-acting agents. This trend has been noticed with inhalational agents, narcotics, benzodiazepines, local anaesthetics and beta blockers. This is in contrast to the trend in other specialities. For example, different needs prompted cardiologists to abandon propranolol for the longer-acting nadolol and atenalol, while anaesthetists are starting to use the shorter-acting esmolol.

This tendency to use short-acting drugs carries the problem of what happens to them. Most drugs are excreted in the urine or the bile or are metabolized, usually in the liver. Thus, kidney and/or liver function is necessary for their disposition. Unfortunately, these organs have limited capacity, considering the short duration of action which we want. The glomeruli in the kidney can filter $1-2 \mathrm{ml} \cdot \mathrm{min}^{-1}$ of plasma per $\mathrm{kg}$ body weight. In the absence of tubular secretion or reabsorption, the clearance $(\mathrm{Cl})$ of a drug totally excreted via the kidney is approximately $1-2 \mathrm{ml} \cdot \mathrm{kg}^{-1} \mathrm{~min}^{-1}$. Then, the elimination half-life $\left(t_{i} \beta\right)$ is directly proportional to the volume of distribution (Vd), according to the formula: ${ }^{1}$

$$
\mathrm{t}_{3} \beta=0.693 \cdot \mathrm{Vd} / \mathrm{Cl} \text {. }
$$

An example of a drug excreted via the kidney is gallamine, which has a relatively small volume of distribution $\left(260 \mathrm{ml} \cdot \mathrm{kg}^{-1}\right){ }^{2}$ In this case, calculations indicate that

$$
\begin{aligned}
\mathrm{t}_{\beta} \beta & =0.693 \cdot 260 \mathrm{ml} \cdot \mathrm{kg}^{-1} / 1.5 \mathrm{ml} \cdot \mathrm{kg}^{-1} \mathrm{~min}^{-1} \\
& =120 \mathrm{~min},
\end{aligned}
$$

which is at the lower limit of the range of values determined experimentally for the drug (128-248 min). ${ }^{2}$ Lipid soluble drugs are handled even less efficiently by

Departments of Anesthesia, Royal Victoria Hospital, \& McGill University, Montreal, Que. the kidney, because they undergo tubular reabsorption and have a larger volume of distribution. Thus, elimination via the kidney is a process which is too inefficient for drugs used in anaesthesia.

In this respect, the liver can do better. Plasma flow through the organ is approximately $10-15 \mathrm{ml} \cdot \mathrm{kg}^{-1}$. $\min ^{-1} .1$ Thus, if gallamine depended entirely on the liver for its disposition, and if this process were 100 per cent efficient, its half-life would be

$$
\begin{aligned}
\mathrm{t} \beta & =0.693 \times 260 \mathrm{ml} \cdot \mathrm{kg}^{-1} / 12 \mathrm{ml} \cdot \mathrm{kg}^{-1} \cdot \mathrm{min}^{-1}, \\
& =15 \mathrm{~min} .
\end{aligned}
$$

The half-life of a lipid soluble drug would be greater. For example, thiopentone has a volume of distribution of 2-3 $\mathrm{L} \cdot \mathrm{kg}^{-1} \cdot{ }^{3}$ Calculations indicate that the liver could not account for an elimination half-life of less than two hours. Because hepatic handling of thiopentone is not 100 per cent efficient, elimination half-lives as high as 12 hours have been reported. ${ }^{3}$

Fortunately, the duration of action of a drug does not necessarily depend on elimination half-life. Redistribution is an important process for most intravenous drugs. A classic example of this is of thiopentone, ${ }^{3}$ which first affects the central nervous system and then is redistributed to fat. Excretion occurs via a relatively slow process when plasma concentrations are much lower than the threshold for pharmacological effect. If redistribution takes place, cumulation may occur if administration of the drug is repeated. However, because the duration of action of the anaesthetic is time-limited, cumulation can usually be avoided and redistribution appears as a useful way to terminate the action of drugs.

Normally, muscle relaxants depend on metabolism or elimination for termination of neuromuscular blockade. Vecuronium, which depends on redistribution, is probably the most important exception. ${ }^{4}$ Longer-acting relaxants all depend on the kidney for a large part of their elimination. As mentioned previously, this dependence on renal function implies a half-life of 1.5-2 hours, a figure which is verified in practice. ${ }^{2}$ For shorter-acting drugs, other mechanisms must be invoked. For succinyl- 
choline, degradation of the drug occurs by enzymatic hydrolysis of the drug in the plasma. This appears as a smart way to overcome the shortcomings of renal and hepatic elimination. As a result of the efficient plasma cholinesterase activity, the half-life of the drug is very short, perhaps $2-4 \mathrm{~min}$, by indirect estimates. ${ }^{5}$ For atracurium, most of the evidence suggests that degradation of the molecule in the plasma is the most important mechanism for the termination of action, ${ }^{6-9}$ although some controversy exists. ${ }^{10}$ Nevertheless, degradation in the plasma or the liver involves the formation of metabolites, whose importance depends on the amount of parent drug administered.

Metabolites can play a significant pharmacological role in several ways. Firstly, they can have an effect similar to that of the parent drug. For example, metabolites of pancuronium ${ }^{11}$ and succinylcholine ${ }^{12}$ are weak neuromuscular blockers. Secondly, they can have undesired side-effects of their own. Laudanosine, a product of atracurium degradation, can cause seizures at high doses. ${ }^{13}$ Thirdly, they can have a toxic, i.e., a dose- and time-related adverse effect. To minimize the effects of metabolites, three strategies are helpful: (1) to use potent drugs which produce small amounts of metabolites; (2) to administer long-acting agents, to avoid the need for numerous top-up doses; and (3) to limit the duration of drug administration. Unfortunately, other considerations may make these strategies unworkable. For neuromuscular blockers, there is evidence that rapid onset can be achieved only with less potent drugs. ${ }^{14}$ A short duration of action is also desired, because a drug is safer if neuromuscular function can be restored rapidly, either at the end of a short procedure or in case of failed tracheal intubation. Finally, such a drug may be required for long procedures or in the intensive care unit. Thus, the role of metabolites of short-acting neuromuscular relaxants needs to be evaluated carefully.

The effect of succinylmonocholine, a breakdown product of succinylcholine, has been studied poorly. Much more attention has been given to atracurium metabolites, ${ }^{15-1}$ but some uncertainties still persist regarding the breakdown of the drug. For example, when atracurium was introduced into clinical practice, Hofmann degradation was considered to be the main route of inactivation. ${ }^{6}$ Then, substantial evidence suggested that ester hydrolysis accounted for as much as two-thirds of the breakdown of the drug. ${ }^{7-9}$ It has also been suggested that organ elimination (probably liver) accounted for at least 50 per cent of the elimination of the drug. ${ }^{10}$ However, this conclusion was derived from a pharmacokinetic study which depends heavily on the validity of several assumptions. At present there is no direct evidence of substantial organ-based elimination for atracurium.
In this issue of the Journal, Nigrovic et al. ${ }^{18}$ report the results of in vitro toxicology studies of atracurium and its metabolites. As they point out, extrapolation to the in vivo human situation should be made with extreme caution because (1) the preparation they used is less viable than human subjects under anaesthesia; (2) the concentrations used were many orders of magnitude greater than those encountered clinically (up to $800 \mu \mathrm{M}$ atracurium compared with less than $0.5 \mu \mathrm{M}$ required for 90 per cent block); and (3) the human body can rely on many scavenging molecules, such as cysteine in Nigrovic et al. 's work. ${ }^{18}$ Still, a few lessons can be learned regarding the use of short-acting drugs which undergo rapid degradation. Firstly, if metabolism occurs solely in plasma, metabolites can be tested relatively easily: incubation in plasma will produce the desired quantity. Secondly, rapidly degraded drugs produce large amounts of metabolites which may be eliminated much more slowly than the parent drug. Thirdly, the effect of these metabolites should be considered seriously whenever prolonged administration occurs. As a result, it appears logical to restrict the use of drugs which are metabolized or degraded rapidly in plasma to short procedures during which a small total dose is given. The use of these agents for prolonged infusions lasting many hours or days may be hazardous, as high levels of metabolites may be attained. Although no direct clinical evidence of atracurium toxicity has been demonstrated in humans, ${ }^{19}$ the possibility should be kept in mind, considering that the administration of longer-acting non-depolarizing relaxants constitutes a suitable and less costly alternative to prolonged infusions of short-acting drugs. More caution should be exercised in the case of future short-acting drugs, such as mivacurium, ${ }^{20,21}$ for which a thorough evaluation of metabolites should be made before it is recommended for prolonged infusions.

\section{L'atracurium, sa pharmacocinétique et ses métabolites}

En anesthésie, on fait appel à une pharmacologie qui se démarque souvent de celle qui s'applique aux autres spécialités. On favorise l'utilisation d'agents particuliers, à action courte, d'utilisation souple. De plus, on ne se préccupe pas outre mesure des effets toxiques, puisque 
la durée d'exposition aux agents est généralement courte. Ainsi, la recherche pharmaceutique s'est consacrée à la découverte d'agents à courte durée d'action. On a pu observer une telle tendance avec les halogénés, les morphiniques, les benzodiazépines, les anesthésiques locaux et les bêta bloquants. Dans d'autres domaines, on a vu une tendance inverse. Par exemple, les cardiologues préfèrent le nadolol et l'aténalol, des agents d'une durée d'action plus longue que celle du propranolol, tandis que l'esmolol, un bêta bloquant à action très courte, se taille une place en anesthésie.

En optant pour des agents à action courte, on doit faire face au problème de leur disposition. La plupart des médicaments sont excrétés dans l'urine et/ou dans la bile, ou encore métabolisess, habituellement au niveau du foie. On a donc besoin d'une fonction hépatique et/ou rénale normale. Ces organes ont toutefois une capacité limitée, si l'on pense à la courte durée d'action que l'on vise. Le rein peut filtrer de 1 à $2 \mathrm{ml} \cdot \mathrm{min}^{-1}$ de plasma par $\mathrm{kg}$ de poids corporel. Il s'ensuit que la clairance (Cl) d'un produit qui n'est ni absorbé ni sécrété dans les tubules est de 1 à $2 \mathrm{ml} \cdot \mathrm{kg}^{-1} \cdot \mathrm{min}^{-1}$. La demi-vie d'élimination $\left(t_{i} \beta\right)$ est alors proportionnelle au volume de distribution (Vd) selon la formule:

$\mathrm{t} \beta=0,693 \cdot \mathrm{Vd} / \mathrm{Cl}$

Par exemple, la gallamine est excrétée par le rein. Elle a aussi un volume de distribution resteint $\left(260 \mathrm{ml} \cdot \mathrm{kg}^{-1}\right){ }^{2}$ Dans ce cas, les calculs donnent:

$$
\begin{aligned}
\mathrm{t}_{\mathrm{i}} \beta & =0,693 \cdot 260 \mathrm{ml} \cdot \mathrm{kg}^{-1} / 1.5 \mathrm{ml} \cdot \mathrm{kg}^{-1} \cdot / \mathrm{min}^{-1} \\
& =120 \mathrm{~min},
\end{aligned}
$$

une valeur quelque peu inférieure à celles qui ont été mesurées expérimentalement (128-248 min). ${ }^{2}$ Le rein se débarrasse encore moins bien des agents liposolubles, qui, en général, sont réabsorbés et ont un grand volume de distribution. Ainsi, on ne peut compter sur le rein pour éliminer les produits utilisés en anesthésie.

A cet égard, le foie peut être plus efficace, parce qu'il reçoit un débit plasmatique de 10 à $15 \mathrm{ml} \cdot \mathrm{kg}^{-1} \cdot \mathrm{min}^{-1}$. Ainsi, si la gallamine était éliminée que par le foie et si ce processus était efficace à 100 pour cent, sa demi-vie serait

$$
\begin{aligned}
4 \beta & =0,693 \cdot 260 \mathrm{ml} \cdot \mathrm{kg}^{-1} / 12 \mathrm{ml} \cdot \mathrm{kg}^{-1} \cdot \mathrm{min}^{-1} \\
& =15 \mathrm{~min} .
\end{aligned}
$$

Dans le cas d'un médicament liposoluble, la demi-vie serait encore plus grande. Par exemple, le thiopental a un volume de distribution de 2 a $3 \mathrm{~L} \cdot \mathrm{kg}^{-1}$. On peut alors calculer qu'avec une activité hépatique intense, la demivie ne peut être plus courte que deux $h$. En fait, la fonction hépatique n'est pas efficace à 100 pour cent de sorte que l'on observe des demi-vies de l'ordre de $12 \mathrm{~h}^{3}$

Toutefois, la durée d'action ne dépend pas toujours de la demi-vie d'élimination. Pour la plupart des agents intraveineux, la redistribution est un processus d'importance capitale. L'exemple le mieux connu de ce phénomène est celui du thiopental, qui est redistribué dans les graisses après avoir agi sur le système nerveux central. L'excrétion devient alors un processus relativement lent qui devient prédominant seulement lorsque les concentrations plasmatiques diminuent sous le seuil correspondant à un effet pharmacologique. S'il y a redistribution, une accumulation peut se produire à la suite d'administrations répétées. Par contre, vu la durée limitée de l'anesthésie, on peut habituellement éviter ce problème et profiter de la redistribution pour mettre fin à l'effet des agents administrés.

Pour mettre fin à la curarisation, on se fie surtout à l'excrétion ou au métabolisme. Le vécuronium, dont la redistribution est importante, est une exception. ${ }^{4}$ Dans le cas des curares à action longue, une partie importante de la dose administrée est éliminée par voie rénale. Tel qu'expliqué plus haut, ceci se traduit par une demi-vie de 1.5 à $2 \mathrm{~h}$, un chiffre qui correspond au données expérimentales. ${ }^{2}$ Pour des agents à durée d'action plus courte, on doit avoir recours à d'autres mécanismes. Par exemple, la succinylcholine est hydrolysee dans le plasma, ce qui constitue une façon élégante de contourner les lenteurs des processus rénal et hépatique. En raison de la grande efficacité de la cholinestérase plasmatique, la demi-vie de la succinylcholine est très courte, soit de deux à quatre minutes d'après une méthode indirecte. ${ }^{5}$ Quant à l'atracurium, sa dégradation plasmatique est tout probablement le mecanisme qui contribue le plus à la décurarisation spontanée. ${ }^{6-9}$ Il y a toutefois controverse à ce sujet. ${ }^{10}$ Quoiqu'il en soit, un métabolisme plasmatique ou hépatique donne lieu a la formation de métabolites, dont la concentration depend de la dose de médicament administrée.

Les métabolites peuvent jouer un rôle pharmacologique significatif à plusieurs égards. Premièrement, leur effet peut ressembler à celui du médicament d'origine. Par exemple, les métabolites du pancuronium ${ }^{11}$ et de la succinylcholine ${ }^{12}$ ont un effet curarisant faible. Ensuite, ces métabolites peuvent avoir des effets secondaires qui leur sont propres. C'est le cas de la laudanosine, un dérivé de l'atracurium, qui peut produire des convulsions à forte dose. ${ }^{13}$ Enfin, ces produits peuvent être toxiques, c'est-à-dire que leur effet néfaste dépend de la dose ainsi que du temps d'exposition. Pour éviter les effets éventuels des métabolites, on peut faire appel à trois stratégies: (1) utiliser des médicaments puissants, qui produisent peu de métabolites; (2) administrer des agents à action prolongée pour éviter de donner des doses additionnelles; et (3) de limiter la durée d'administration. Malheureusement, on doit souvent tenir compte de d'autres facteurs. Pour les 
curares, il semble qu'un délai d'action rapide ne soit possible qu'avec des agents peu puissants. ${ }^{14} \mathrm{Il}$ faut aussi une durée d'action courte parce qu'on veut une décurarisation rapide à la fin d'une intervention courte ou encore si l'intubation endotrachéale s'avère une tâche impossible. Enfin, on aimerait utiliser le même curare pour des interventions longues ou aux soins intensifs. Ainsi, il faut évaluer avec soin les métabolites des curares à action courte.

La succinylmonocholine, un produit de l'hydrolyse de la succinylcholine, n'a pas été bien étudiée. Tel n'est pas le cas des métabolites de l'atracurium. ${ }^{15-17}$ Toutefois, on fait encore face à certaines incertitudes. Par exemple, lorsqu'on a mis l'atracurium sur le marché, on pensait que la dégradation de Hofmann était le mécanisme principal d'inactivation. ${ }^{6}$ Depuis lors, plusieurs données ont permis de croire que l'atracurium est hydrolysée au deux tiers par les estérases plasmatiques. ${ }^{7-9}$ On a aussi émis l'hypothése que plus de 50 pour cent de l'atracurium injectée était éliminée par un orgạne (probablement le foie) ${ }^{10}$ On est arrivé à cette conclusion en se basant sur des données cinétiques dont l'exactitude dépend strictement de la validité des hypothèses de départ. Jusqu'à présent, on n'a donc pas fourni de preuve directe que l'élimination de l'atracurium dépende d'un organe quelconque.

Dans ce numéro du Journal, Nigrovic et coll. ${ }^{18}$ décrivent des expériences sur la toxicologie de l'atracurium et de ses métabolites, in vitro. Comme les auteurs l'admettent eux-mêmes, il faut extrapoler ces résultats aux humains avec une extrême prudence, parce que (1) la préparation utilisée est moins viable que des sujets humains sous anesthesie, (2) les concentrations dont on fait état dans l'article (jusqu'à $800 \mu \mathrm{M}$ ) dépassent de plusieurs ordres de grandeur celles qu'on obtient en clinique (environ $0.5 \mu \mathrm{M}$ pour un bloc de 90 pour cent), et (3) il existe des mécanismes de détoxification, un rôle joú́ par la cystéine dans le travail de Nigrovic et coll..$^{18}$ Toutefois, on peut tirer des conclusions utiles au sujet des agents à action courte qui sont dégradés rapidement. Tout d'abord, si le métabolisme est exclusivement plasmatique, on peut étudier les métabolites assez facilement: une incubation dans le plasma nous en fournit en quantité voulue. Ensuite, il faut tenir compte du fait que les médicaments qui sont dégradés rapidement produisent de grandes quantités de métabolites, et que ceux-ci sont souvent éliminés lentement. Enfin, on doit se préoccuper de l'effet de ces métabolites lorsqu'on a une administration prolongée. Conséquemment, il semble logique de restreindre l'utilisation de médicaments qui sont dégradés rapidement aux interventions courtes, pendant lesquelles on n'administre qu'une petite quantité de ces produits. $\mathrm{Si}$ on les injecte sous forme de perfusion pour des périodes allant de plusieurs heures à plusieurs jours, on pourrait atteindre des concentrations dangereuses de métabolites. Chez les humains, on n'a aucune preuve de la toxicité de l'atracurium donné dans ces conditions, ${ }^{19}$ mais on doit tenir compte de cette possibilite, puisqu'on peut remplacer, à coût avantageux, l'atracurium par des curares à action prolongee. En particulier, on devrait appliquer cette consigne de prudence à tous les curares à action courte qui pourraient devenir disponibles dans l'avenir, dont le mivacurium. ${ }^{20.21}$ Dans ce cas, on devrait s'abstenir de recommander ces curares pour des perfusions de longue durée sans étude approfondie des métabolites.

\section{References}

1 Wilkinson GR, Shand DG. A physiological approach to the hepatic drug clearance. Clin Pharmacol Ther 1975; 18: $377-90$.

2 Shanks $C A$. Pharmacokinetics of the nondepolarizing neuromuscular relaxants applied to the calculation of bolus and infusion dosage regimens. Anesthesiology 1986; 64: 72-86.

3 Burch $P G$, Stanski $D R$. The role of metabolism and protein binding in thiopental anesthesia. Anesthesiology 1983; 58: $146-52$.

4 Sohn YJ, Bencini AF. Scaf AHJ, Kersten UW, Agoston $S$. Comparative pharmacokinetics and dynamics of vecuronium and pancuronium in anesthetized patients. Anesth Analg 1986; 65: 233-9.

5 Cook $D R$, Wingard LB, Taylor FH. Pharmacokinetics of succinylcholine in infants, children and adults. Clin Pharmacol Ther 1976; 20: 493-8.

6 Merrett RA, Thompson $C W$, Webb FW. In vitro degradation of atracurium in human plasma. $\mathrm{Br} J$ Anaesth 1983; 55: 61-6.

7 Stiller RL, CookDR, Chakravorti S. In vitro degradation of atracurium in human plasma. $\mathrm{Br} J$ Anaesth $1985 ; 1085-8$.

8 Ward S, Boheimer N, Weatherley BC, Simmonds RJ, Dopson TA. Pharmacokinetics of atracurium and its metabolites in patients with normal renal function, and in patients in renal failure. $\mathrm{Br} J$ Anaesth 1987; 59: 697-706.

9 Tsui D. Graham GG, Torda TA. The pharmacokinetics of atracurium isomers in vitro and in humans. Anesthesiology 1987; 67: 722-8.

10 Fisher DM, Canfell PC, Fahey MR et al. Elimination of atracurium in humans: contribution of Hofmann elimination and ester hydrolysis versus organ-based elimination. Anesthesiology 1986; 65: 6-12.

11 Miller RD, Agoston S, Booij LHD, Kersten UW, Crul JF, Ham J. The comparative potency and pharmacokinetics of pancuronium and its metabolites in anesthetized man. $J$ Pharmacol Exp Ther 1978; 207: 539-43. 
12 Brennan HJ. Dual action of suxamethonium chloride. Br J Anaesth 1956; 28: 159-68.

13 Chapple DJ, Miller AA, Ward JB, Wheatley PL. Cardiovascular and neurological effects of laudanosine. Studies in mice and rats, and in conscious and anaesthetized dogs. $\mathrm{Br} \mathbf{J}$ Anaesth 1987; 59: 218-25.

14 Bowman WC, Rodger $M$, Houston J, Marshall RJ, McIndewar I. Structure:action relationships among some desacetoxy analogues of pancuronium and vecuronium in the anesthetized cat. Anesthesiology 1988; 69: 57-62.

15 Nigrovic $V$, Pandya $J B$, Auen $M$, Wajskol $A$. Inactivation of atracurium in human and rat plasma. Anesth Analg 1985; 64: 1047-52.

16 Nigrovic V, Klaunig JE, Smith SL, Schuitz NE, Wajskol $A$. Comparative toxicity of atracurium and metocurine in isolated rat hepatocytes. Anesth Analg 1986; 65: 1107-11.

17 Nigrovic $V$, Smith $S$. Involvement of nucleophiles in the inactivation of atracurium. Br J Anaesth 1987; 59: 617-21.

18 Nigrovic $V$, Pandya JB, Klaunig JE, Fry K. Reactivity and toxicity of atracurium and its metabolites in vitro. Can $\mathrm{J}$ Anaesth 1989; 36: 262-8 (current journal).

19 Parker CJR, Jones JE, HunterJM. Disposition of infusions of atacurium and its metabolite, laudanosine, in patients in renal and respiratory failure in an ITU. Br J Anaesth 1988; 61: $531-40$.

20 Weber S, Brandom BW, Powers DM et al. Mivacurium chloride (BW I090U)-induced neuromuscular blockade during nitrous oxide-narcotic anesthesia in adult surgical patients. Anesth Analg 1988; 67: 495-9.

21 Savarese JJ, Ali HH, Basta SJ et al. The clinical neuromuscular pharmacology of mivacurium chloride (BW 1090U). Anesthesiology 1988; 68: 723-32. 